\title{
Multiple Comparison of Medians Using Permutation Tests
}

Scott J. Richter

University of North Carolina at Greensboro, sjicht2@uncg.edu

Melinda H. McCann

Oklahoma State University

Follow this and additional works at: http://digitalcommons.wayne.edu/jmasm

Part of the Applied Statistics Commons, Social and Behavioral Sciences Commons, and the Statistical Theory Commons

\section{Recommended Citation}

Richter, Scott J. and McCann, Melinda H. (2007) "Multiple Comparison of Medians Using Permutation Tests," Journal of Modern Applied Statistical Methods: Vol. 6 : Iss. 2 , Article 6.

DOI: $10.22237 /$ jmasm/1193889900

Available at: http://digitalcommons.wayne.edu/jmasm/vol6/iss2/6 


\section{Multiple Comparison Of Medians Using Permutation Tests}

\author{
Scott J. Richter \\ University of North Carolina at Greensboro
}

\author{
Melinda H. McCann \\ Oklahoma State University
}

A robust method is proposed for simultaneous pairwise comparison using permutation tests and median differences. The new procedure provides strong control of familywise error rate and has better power properties than the median procedure of Nemenyi/Levy. It can be more powerful than the Tukey-Kramer procedure using mean differences, especially for nonnormal distributions and unequal sample sizes.

Key words: Simultaneous inference, pairwise comparisons, median difference, permutation test.

\section{Introduction}

The technique of using permutation methods for multiple comparisons has received relatively little attention in the literature. Nemenyi (1963) and later Levy (1979) proposed a procedure using medians, with the maximum of the differences of pairwise Mood statistics used to construct the reference distribution. Miller (1966, 1981), and more recently Higgins (2004), proposed a permutation version of the TukeyKramer method (Tukey, 1949; Kramer, 1956), where the range of the sample means is calculated for each permutation of observations among the $k$ groups to obtain the reference distribution. The mean difference for each pair of means is then compared to this reference distribution to determine statistically significant differences. However, when distributions are skewed or there are outliers in the data, it may be desirable to make comparisons of medians rather than means. Thus, a logical extension of Miller's procedure is to replace means by medians. Consider the following example.

Scott J. Richter is Associate Professor and Director of the Statistical Consulting Center. His research interests are in robust methods and applied statistics. Email him at sjricht2@uncg.edu. Melinda McCann is Associate Professor of Statistics. Her research interests involve multiple comparisons procedures and their applications.
Example

Manly (1997) reported the data in Table 1 based on articles by Powell \& Russell (1984, 1985) and Linton et al (1989). The data represent dry biomass (in $\mathrm{mg}$ ) of ants for 24 eastern horned lizards, taken in three months in 1980.

It is desired to determine which, if any, of the months have different consumptions. The relation between the means and medians for each month suggests that the distributions of biomass are skewed, and that the means may not be representative of monthly consumption. Thus, comparisons based on medians may be more appropriate.

Both the median procedure of Nemenyi and Levy and Miller's procedure permute freely across all groups (unrestricted randomization). However, this unrestricted randomization scheme has been criticized. Petrondas and Gabriel (1983) contend that Miller's approach does not control the familywise error rate (FWE): the probability of making at least one false declaration of inequality, since the test for any subset hypothesis that a pair of means is equal should be based on permuting observations only among the groups whose distributions are assumed equal under the null hypothesis. The FWE actually is controlled under the overall null hypothesis that all $k$ distributions have the same location - that is, in the weak sense (Hochberg \& Tamhane, 1987), but not necessarily under a subset pairwise null hypothesis that requires only the two distributions being considered to have equal 
Table 1. Dry biomass of ants for 24 eastern horned lizards, taken in three months in 1980.

$\begin{array}{cccl}\text { Month } & \text { Dry biomass }(\mathrm{mg}) & \text { Median } & \text { Mean } \\ \text { June } & 13,242,105 & 105.0 & 120.0 \\ \text { July } & 8,59,20,2,245 & 20.0 & 66.8 \\ \text { August } & 515,488,88,233,50,600,82,40,52,1889 & 160.5 & 403.7\end{array}$

location, that is, in the strong sense (Hochberg \& Tamhane, 1987). Accordingly, both Petrondas and Gabriel (1983) and Hochberg and Tamhane (1987) suggest performing each pairwise test separately using a Bonferroni adjustment. Similarly, Hochberg and Tamhane (1987) and Ryan and Ryan (1980) note that the median procedure of Nemenyi/Levy is not based on a joint testing family, and thus does not control the FWE. Hochberg and Tamhane (1987) instead suggest permuting separately within each pair (restricted randomization) and utilizing the maximum of pairwise Mood statistics to derive the reference distribution.

A new testing procedure is proposed based on the procedure of Nemenyi/Levy, using median difference statistics instead of differences between Mood statistics, and Type I error and power properties are compared to the new procedure to those of the Nemenyi/Levy procedure, pairwise tests using a Bonferroni adjustment, and also to the Tukey-Kramer procedure based on mean differences, which assumes normally distributed populations.

\section{Methodology}

Throughout, consider a one-way layout with $k$ groups, where $F_{i}$ is the common continuous distribution function for the $i^{\text {th }}$ group, $n_{i}$ is the sample size of the $i^{\text {th }}$ group, and $N=n_{1}+n_{2}+\cdots+n_{k}$. Further, let $\mu_{i}$ be the location parameter associated with the $i^{\text {th }}$ distribution and $\hat{\mu}_{i}$ be the sample median for the $i^{\text {th }}$ group. Distributions are assumed identical for all treatments except for possible location differences.
Permutation-based Multiple Comparison Procedures:

Miller (1966, 1981) proposed a permutation analog to the Tukey-Kramer procedure for multiple pairwise comparison of several means. The reference distribution for Miller's method was based on the statistic, $\max _{1 \leq i<j \leq k}\left|\bar{Y}_{i}-\bar{Y}_{j}\right|$, where $\bar{Y}_{i}$ and $\bar{Y}_{j}$ are the respective sample means of groups $i$ and $j$. The reference distribution consists of the values of this statistic for all $\frac{N !}{n_{1} ! n_{2} ! \cdots n_{k} !}$ possible permutations of the observed data. Each pairwise absolute difference is compared to this distribution to determine statistical significance. Bonferroni-adjusted pairwise tests suggested by Hochberg and Tamhane (1987) and Petrondas and Gabriel (1983) will also be considered.

Nemenyi (1963) and later Levy (1979) also proposed an analog to the Tukey-Kramer procedure, but based on Mood's (1950) median test, as follows. First, calculate the grand median for the pooled sample of $N=n_{1}+n_{2}+\cdots+n_{k}$ observations. Then determine $M_{i}$, the number of observations in the $i^{\text {th }}$ sample that exceed the grand median. The test statistic for comparing any pair is $\left|\frac{M_{i}}{n_{i}}-\frac{M_{j}}{n_{j}}\right|$. The reference distribution is based on the distribution of $\max _{1 \leq i<j \leq k}\left|\frac{M_{i}}{n_{i}}-\frac{M_{j}}{n_{j}}\right|$, the maximum value of the test statistic over all pairs, which is calculated for a large set of random reassignments of observations to groups. As with Miller's method, an observation may be 
reassigned to any of the $k$ groups to form a new permutation. Hochberg and Tamhane (1987) suggest computing a separate grand median for each pair and calculating the test statistic above. The maximum over all pairs is then found for a large set of random reassignments, where reassignments are restricted to within each pair, and these values form the reference distribution.

\section{A New Method Using Median Differences:}

In situations involving skewed distributions or outliers it may be more appropriate to consider medians instead of means. Thus, we propose multiple comparison procedures based on median differences. The method of Nemenyi/Levy, based on Mood statistics, does utilize medians, but does not incorporate the magnitude of the difference between medians. It is believed that there may be situations when incorporating this information could lead to a more sensitive procedure.

Analogous to the mean-based procedure of Miller, the reference distribution for our new procedure is based on the distribution of $\max _{1 \leq i<j \leq k}\left|\hat{\mu}_{i}-\hat{\mu}_{j}\right|$, the maximum of all pairwise median differences, calculated for a large set of random reassignments of observations to groups. Each pairwise absolute median difference is compared to this reference distribution to determine statistical significance. Both methods of permuting discussed in Section 2.1, namely restricted and unrestricted, are investigated.

Restricted Randomization Guarantees FWE Control:

The strongest argument against unrestricted permuting is that it does not necessarily provide strong control of the FWE. Restricted permuting, however, does provide strong control.

Consider $k$ independent samples from distributions that differ by at most a location parameter. That is, for $i, j=1,2, \ldots, k$ with $i<j$, $F_{i}(x)=F_{j}\left(x-\Delta_{i j}\right)$. (Throughout Section 2.3 let $i, j=1,2, \ldots, k$ with $i<j$.) $\quad$ The null hypothesis then involves $\left(\begin{array}{l}k \\ 2\end{array}\right)$ pairwise hypotheses of the form $H_{0 i j}: \Delta_{i j}=0$. Now consider the permutation distribution of median differences from samples $i$ and $j$, and let $D_{i j}(\alpha)$ be the $1-\alpha$ percentile of this permutation distribution. Similarly, define $D_{\max }(\alpha)$ to be the $1-\alpha$ percentile of the permutation distribution for the maximum median difference among all $\left(\begin{array}{l}k \\ 2\end{array}\right)$ pairs.

First consider the case under the complete null hypothesis where all $\Delta_{i j}=0$. Let the calculated median difference from samples $i$ and $j$ be denoted by $\tilde{D}_{i j}$. Under the complete null hypothesis the probability that a calculated median difference from a particular pair of samples in a given permutation is the maximum difference is $\left(\begin{array}{l}k \\ 2\end{array}\right)^{-1}$. Thus, each pair of samples will contribute $\alpha\left(\begin{array}{l}k \\ 2\end{array}\right)^{-1}$ of the values from the pairwise difference permutation distribution to the maximum difference permutation distribution.

Consequently, the probability that any observed difference from a particular pair exceeds $D_{\max }(\alpha)$, the comparisonwise error rate, is $\alpha\left(\begin{array}{l}k \\ 2\end{array}\right)^{-1}$. Alternatively, the familywise error rate is given by

$P($ declare at least one pair different in location | all pairs have equal location)

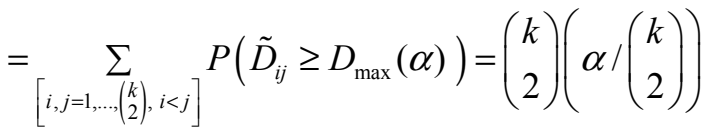

$$
\begin{aligned}
& =\alpha \text {. }
\end{aligned}
$$

This shows that using the permutation distribution of the maximum difference controls 
the FWE in the weak sense (Hochberg \& Tamhane, 1987).

Now consider the case where only $t<\left(\begin{array}{l}k \\ 2\end{array}\right)$ of the pairwise null hypotheses are indeed true. For any permutation, a difference from one of these $t$ pairs with a true pairwise null hypothesis is less likely to be the maximum difference than differences from the $\left(\begin{array}{l}k \\ 2\end{array}\right)-t$ pairs where $\Delta_{i j} \neq 0$. Consequently, the comparisonwise error rate is $P\left(\tilde{D}_{i j} \geq D_{\max }(\alpha)\right) \leq \alpha\left(\begin{array}{l}k \\ 2\end{array}\right)^{-1}$. Thus, the familywise error rate, the probability of rejecting at least one of the $t$ true null hypotheses, is

$P($ reject at least one true null hypothesis $\mid$ $t$ true null hypotheses $) \leq t\left(\alpha /\left(\begin{array}{l}k \\ 2\end{array}\right)\right)<\alpha$.

Thus, the FWE is controlled at level $\alpha$ for any combination of $t$ true and $\left(\begin{array}{l}k \\ 2\end{array}\right)-t$ false hypotheses, and the FWE is controlled in the strong sense (Hochberg \& Tamhane, 1987).

Alternatively, the FWE may be controlled by performing separate two-sample permutation tests and utilizing $\alpha\left(\begin{array}{l}k \\ 2\end{array}\right)^{-1}$, a Bonferroni adjustment, as the significance level for each individual comparison. Based on their performance in the normal theory setting, it is expected that a Tukey-type permutation procedure will generally be less conservative than a procedure utilizing pairwise permutation tests with a Bonferroni adjustment.

\section{Simulation Study}

A simulation was conducted to evaluate five permutation procedures:

1. A modification of Miller's $(1966,1981)$ procedure, using medians instead of means and unrestricted randomization (MEDUR);

2. A modification of (1) using restricted randomization (MEDR);

3. Separate Bonferroni-adjusted pairwise permutation tests for median differences (MEDBON);

4. The procedure of Nemenyi (1963)/Levy (1979) based on differences between Mood statistics and unrestricted randomization (MOODUR);

5. A modification of (4), using restricted randomization (MOODR).

The following model was assumed to generate the data:

$$
y_{i j}=\mu_{i}+e_{i j},
$$

where $y_{i j}=$ the $j^{\text {th }}$ observation for the $i^{\text {th }}$ treatment $\mu_{i}=$ the location parameter for the $i^{\text {th }}$ treatment $e_{i j}=$ the random error associated with the $j^{\text {th }}$ observation for the $i^{\text {th }}$ treatment. The $e_{i j}$ are assumed independent and identically distributed.

Several different error distributions were examined:

- $\operatorname{Normal}\left(\mu=0, \sigma^{2}=1\right)$;

- Uniform $[-3,3]$

- Exponential $(\lambda=3)$;

- Double exponential $(\operatorname{Exp}(\lambda=3)-E$ $\operatorname{xp}(\lambda=3))$

- Location-contaminated normal $(N(0,1)$ with $10 \%$ contamination from $N(9,1))$.

These choices encompass two symmetric, nonnormal distributions: the uniform (lightertailed than normal) and the double exponential (heavier-tailed than normal); and two skewed distributions: the exponential and contaminated normal. Models contained either three or five groups, and both equal and unequal sample sizes were examined. In most cases the total number of permutations possible is prohibitive, and thus a random sample of permutations was used to estimate the $p$-value for any given test. KellerMcNulty and Higgins (1987) examined the issue 
of randomly sampling the permutations, and concluded that little is to be gained by taking more than 1600 randomly sampled permutations. Thus, each permutation test was based on a reference distribution estimated via a slightly conservative 2000 randomly sampled permutations, and the estimated proportions of rejections were based on 2000 randomly generated samples. The simulations were implemented using Resampling Stats version 5.0 (Resampling Stats Inc., 2000).

The familywise error rate (FWE) and any-pair power (Shaffer, 1995), the probability of detecting at least one true difference, are reported in the Tables 2-12. For the Tukey-type procedures based on medians, in cases where either all groups have identical locations or all groups had different locations, these were estimated by comparing the maximum pairwise difference from among the samples to the respective reference distribution, and counting the number of random samples where this maximum was in the top $5 \%$ of the reference distribution. In cases where some pairs had identical locations while others pairs differed in location, the FWE was estimated as the proportion of permutations where at least one of the true null hypotheses was rejected (strong FWE).

\section{Results}

\section{Comparison of Median-based Procedures}

Type I Error

All median-based procedures controlled the FWE in the strong sense (See Tables 2-4). In fact, in the cases where some pairs had equal locations and some did not, the probability of at least one false rejection was usually lower than the case where all locations were equal. As Petrondas and Gabriel (1983) admitted, their counterexample was very small, and, "for realistic, larger examples the corresponding tests (using unrestricted permuting) may be both valid and useful." It is also worth noting, however, that even though the unrestricted permuting method did not exhibit inflated FWE rates for either the median difference statistic or the Mood statistic, in cases where there was a difference between unrestricted and restricted FWE rates, the unrestricted FWE was almost always higher. This was true especially with unequal sample sizes, where error rates more than twice as large for unrestricted permuting were not uncommon. As we shall see in the next section, however, higher FWE rates did not typically lead to more powerful tests. In light of this evidence and the earlier cited criticisms of unrestricted randomization, as well as the fact that power is generally at least as good under restricted randomization, only procedures using restricted randomization will be considered in the remainder of the discussion.

Power

Consider first the case of equal sample sizes. With small group sample size $(n=5)$ and small location differences $\left(\Delta_{1}=\Delta_{2}=0, \Delta_{3}=2\right.$ or $\left.\Delta_{1}=\Delta_{2}=2, \Delta_{3}=\Delta_{4}=\Delta_{5}=0\right)$, MEDR always had the highest power among the median procedures (See Tables 5 and 7). When there were larger location differences ( $\Delta_{1}=\Delta_{2}=2, \Delta_{3}=5$ or $\Delta_{1}=\Delta_{2}=2, \Delta_{3}$ $=3, \Delta_{4}=\Delta_{5}=0$ ),MOODR often had highest power for normal and contaminated normal data (e.g., see Table 6). On the other hand, MEDBON had no power with $n=5$ (See Tables 5-7). With group sample size $n=10$ (e.g., see Table 8), MEDR was often most powerful for heavier-tailed distributions (exponential, double exponential), especially with larger location differences and more groups (e.g., 3 groups, $n=10, \Delta_{1}=\Delta_{2}=2, \Delta_{3}=5 ; 5$ groups, $n=10, \Delta_{1}=\Delta_{2}=2, \Delta_{3}=\Delta_{4}=\Delta_{5}=0$ ) while MOODR was most powerful for the latter five group scenarios for contaminated normal data. MEDBON often had higher power than MOODR, but always trailed MEDR. For $n=20$, MEDBON was most powerful for uniform and exponential data, and all three median-based procedures had similar power for the other distributions (See Table 9). MEDR performed most consistently across different scenarios, was never much less powerful than any other procedure for nonnormal data, and was often substantially more powerful. For example, in Table 11, MEDR had power almost 200 times the power of MOODR (0.591 versus 0.003 ), while the largest power advantage for 
Table 2. FWE - Proportion of times at least one true null hypothesis was rejected at $\alpha=0.05$, three groups, $n_{i}=5$, locations $\Delta_{1}=\Delta_{2}=\Delta_{3}=0$.

$\begin{array}{lccccc}\text { Procedure } & \text { Normal } & \text { Uniform } & \begin{array}{c}\text { Distribution } \\ \text { Double-Exp. }\end{array} & \text { Exponential } & \text { Cont.-Normal } \\ \text { MEDR } & 0.053 & 0.046 & 0.047 & 0.037 & 0.027 \\ \text { MEDUR } & 0.035 & 0.041 & 0.054 & 0.040 & 0.019 \\ \text { MOODR } & 0.013 & 0.018 & 0.017 & 0.019 & 0.007 \\ \text { MOODUR } & 0.009 & 0.013 & 0.011 & 0.013 & 0.003 \\ \text { TUKEY } & 0.053 & 0.059 & 0.060 & 0.044 & 0.026\end{array}$

Table 3. FWE - Proportion of times at least one true null hypothesis was rejected at $\alpha=0.05$, five groups, $n_{i}=5$, locations $\Delta_{1}=\Delta_{2}=2 ; \Delta_{3}=\Delta_{4}=\Delta_{5}=0$.

$\begin{array}{lccccc}\text { Procedure } & \text { Normal } & \text { Uniform } & \begin{array}{c}\text { Distribution } \\ \text { Double-Exp. }\end{array} & \text { Exponential } & \text { Cont.-Normal } \\ \text { MEDR } & 0.000 & 0.009 & 0.009 & 0.014 & 0.000 \\ \text { MEDUR } & 0.000 & 0.023 & 0.017 & 0.021 & 0.001 \\ \text { MOODR } & 0.001 & 0.008 & 0.005 & 0.003 & 0.001 \\ \text { MOODUR } & 0.001 & 0.008 & 0.005 & 0.003 & 0.001 \\ \text { TUKEY } & 0.024 & 0.025 & 0.025 & 0.023 & 0.025\end{array}$

Table 4. FWE - Proportion of times at least one true null hypothesis was rejected at $\alpha=0.05$, five groups, $n_{1}=3, n_{2}=4, n_{3}=5, n_{4}=6, n_{5}=7$, locations $\Delta_{1}=\Delta_{2}=2 ; \Delta_{3}=\Delta_{4}=\Delta_{5}=0$.

$\begin{array}{lccccc}\text { Procedure } & \text { Normal } & \text { Uniform } & \begin{array}{c}\text { Distribution } \\ \text { Double-Exp. }\end{array} & \text { Exponential } & \text { Cont.-Normal } \\ \text { MEDR } & 0.001 & 0.005 & 0.008 & 0.006 & 0.003 \\ \text { MEDUR } & 0.003 & 0.013 & 0.025 & 0.014 & 0.026 \\ \text { MOODR } & 0.001 & 0.005 & 0.007 & 0.001 & 0.002 \\ \text { MOODUR } & 0.001 & 0.005 & 0.007 & 0.001 & 0.002 \\ \text { TUKEY } & 0.000 & 0.000 & 0.000 & 0.001 & 0.001\end{array}$


Table 5. Power - Proportion of times at least one pairwise difference detected at $\alpha=0.05$, three groups, $n_{i}=5$, locations $\Delta_{1}=\Delta_{2}=0, \Delta_{3}=2$.

$\begin{array}{lccccc}\text { Procedure } & \text { Normal } & \text { Uniform } & \begin{array}{c}\text { Distribution } \\ \text { Double-Exp. }\end{array} & \text { Exponential } & \text { Cont.-Normal } \\ \text { MEDR } & 0.579 & 0.269 & 0.098 & 0.151 & 0.336 \\ \text { MEDUR } & 0.487 & 0.256 & 0.095 & 0.113 & 0.297 \\ \text { MEDBON } & 0.000 & 0.000 & 0.000 & 0.000 & 0.000 \\ \text { MOODR } & 0.238 & 0.064 & 0.049 & 0.080 & 0.133 \\ \text { MOODUR } & 0.131 & 0.045 & 0.039 & 0.055 & 0.070 \\ \text { TUKEY } & 0.818 & 0.342 & 0.125 & 0.186 & 0.478\end{array}$

Table 6. Power - Proportion of times at least one pairwise difference detected at $\alpha=0.05$, three groups, $n_{i}=5$, locations $\Delta_{1}=0, \Delta_{2}=2, \Delta_{3}=5$.

$\begin{array}{lccccc}\text { Procedure } & \text { Normal } & \text { Uniform } & \begin{array}{c}\text { Distribution } \\ \text { D-Exp }\end{array} & \text { Exponential } & \text { Cont-Normal } \\ \text { MEDR } & 0.786 & 0.707 & 0.262 & 0.410 & 0.455 \\ \text { MEDUR } & 0.976 & 0.716 & 0.220 & 0.422 & 0.581 \\ \text { MEDBON } & 0.000 & 0.000 & 0.000 & 0.000 & 0.000 \\ \text { MOODR } & 0.888 & 0.469 & 0.156 & 0.302 & 0.537 \\ \text { MOODUR } & 0.820 & 0.377 & 0.127 & 0.248 & 0.499 \\ \text { TUKEY } & 1.000 & 0.979 & 0.350 & 0.620 & 0.590\end{array}$

Table 7. Power - Proportion of times at least one pairwise difference detected at $\alpha=0.05$, five groups, $n_{i}=5$, locations $\Delta_{1}=\Delta_{2}=2 ; \Delta_{3}=\Delta_{4}=\Delta_{5}=0$.

$\begin{array}{lccccc}\text { Procedure } & \text { Normal } & \text { Uniform } & \begin{array}{c}\text { Distribution } \\ \text { Double-Exp. }\end{array} & \text { Exponential } & \text { Cont.-Normal } \\ \text { MEDR } & 0.637 & 0.369 & 0.059 & 0.137 & 0.396 \\ \text { MEDUR } & 0.400 & 0.293 & 0.078 & 0.104 & 0.245 \\ \text { MEDBON } & 0.000 & 0.000 & 0.000 & 0.000 & 0.000 \\ \text { MOODR } & 0.477 & 0.112 & 0.096 & 0.135 & 0.303 \\ \text { MOODUR } & 0.477 & 0.112 & 0.096 & 0.135 & 0.303 \\ \text { TUKEY } & 0.886 & 0.422 & 0.000 & 0.186 & 0.540\end{array}$


Table 8. Power - Proportion of times at least one pairwise difference detected at $\alpha=0.05$, three groups, $n_{i}=10$, locations $\Delta_{1}=0, \Delta_{2}=2, \Delta_{3}=5$.

$\begin{array}{lccccc}\text { Procedure } & \text { Normal } & \text { Uniform } & \begin{array}{c}\text { Distribution } \\ \text { Double-Exp. }\end{array} & \text { Exponential } & \text { Cont.-Normal } \\ \text { MEDR } & 1.000 & 0.996 & 0.661 & 0.949 & 0.923 \\ \text { MEDUR } & 1.000 & 0.990 & 0.635 & 0.904 & 0.911 \\ \text { MEDBON } & 1.000 & 1.000 & 0.574 & 0.947 & 0.854 \\ \text { MOODR } & 0.888 & 0.469 & 0.156 & 0.302 & 0.537 \\ \text { MOODUR } & 0.820 & 0.377 & 0.127 & 0.248 & 0.499 \\ \text { TUKEY } & 1.000 & 1.000 & 0.627 & 0.890 & 0.940\end{array}$

Table 9. Power - Proportion of times at least one pairwise difference detected at $\alpha=0.05$, three groups, $n_{i}=20$, locations $\Delta_{1}=\Delta_{2}=0, \Delta_{3}=2$.

$\begin{array}{lccccc}\text { Procedure } & \text { Normal } & \text { Uniform } & \begin{array}{c}\text { Distribution } \\ \text { Double-Exp. }\end{array} & \text { Exponential } & \text { Cont-Normal } \\ \text { MEDR } & 1.000 & 0.664 & 0.374 & 0.664 & 0.991 \\ \text { MEDUR } & 1.000 & 0.676 & 0.361 & 0.676 & 0.979 \\ \text { MEDBON } & 1.000 & 0.776 & 0.342 & 0.776 & 0.983 \\ \text { MOODR } & 0.998 & 0.569 & 0.384 & 0.648 & 0.996 \\ \text { MOODUR } & 0.997 & 0.529 & 0.352 & 0.614 & 0.992 \\ \text { TUKEY } & 1.000 & 0.550 & 0.278 & 0.550 & 0.436\end{array}$

Table 10. Power - Proportion of times at least one pairwise difference detected at $\alpha=0.05$, three groups, $n_{1}=4, n_{2}=5, n_{3}=6$, locations $\Delta_{1}=\Delta_{3}=0, \Delta_{2}=2$.

Procedure

$\begin{array}{llllll}\text { MEDR } & 0.607 & 0.260 & 0.090 & 0.129 & 0.287 \\ \text { MEDUR } & 0.558 & 0.262 & 0.093 & 0.121 & 0.264 \\ \text { MEDBON } & 0.332 & 0.108 & 0.047 & 0.100 & 0.203 \\ \text { MOODR } & 0.147 & 0.041 & 0.060 & 0.070 & 0.125 \\ \text { MOODUR } & 0.147 & 0.041 & 0.060 & 0.070 & 0.125 \\ \text { TUKEY } & 0.220 & 0.035 & 0.005 & 0.012 & 0.051\end{array}$


Table 11. Power - Proportion of times at least one pairwise difference detected at $\alpha=0.05$, three groups, $n_{1}=4, n_{2}=5, n_{3}=6$, normally distributed data.

$\begin{array}{lccc}\text { Location pattern } \\ \text { Procedure } & \Delta_{1}=2, \Delta_{2}=\Delta_{3}=0 & \Delta_{1}=\Delta_{3}=0, \Delta_{2}=2 & \Delta_{1}=\Delta_{2}=0, \Delta_{3}=2 \\ \text { MEDR } & 0.591 & 0.607 & 0.711 \\ \text { MEDUR } & 0.656 & 0.558 & 0.478 \\ \text { MEDBON } & 0.302 & 0.332 & 0.458 \\ \text { MOODR } & 0.003 & 0.147 & 0.654 \\ \text { MOODUR } & 0.003 & 0.147 & 0.654 \\ \text { TUKEY } & 0.219 & 0.220 & 0.228\end{array}$

Table 12. Power - Proportion of times at least one difference detected at $\alpha=0.05$, five groups, $n_{1}=3, n_{2}=4, n_{3}=5, n_{4}=6, n_{5}=7$, normally distributed data.

$$
\begin{aligned}
& \text { Location pattern }
\end{aligned}
$$

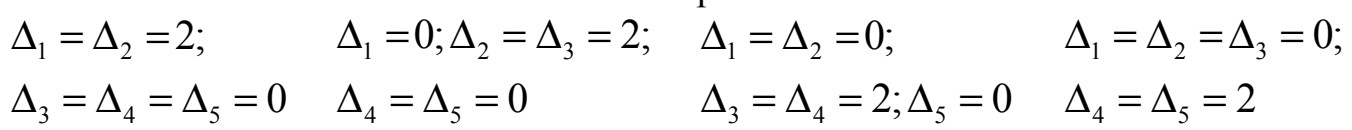

Procedure

$\begin{array}{lllll}\text { MEDR } & 0.546 & 0.451 & 0.556 & 0.702 \\ \text { MEDUR } & 0.516 & 0.372 & 0.322 & 0.298 \\ \text { MEDBON } & 0.003 & 0.000 & 0.041 & 0.002 \\ \text { MOODR } & 0.001 & 0.001 & 0.416 & 0.832 \\ \text { MOODUR } & 0.001 & 0.001 & 0.430 & 0.831 \\ \text { TUKEY } & 0.000 & 0.032 & 0.025 & 0.024\end{array}$

MOODR was less than 1.2 times that of MEDR, 0.537 versus 0.455 See Table 6). Table 8 shows, however, that when the sample size increased from $n=5$ to $n=10$, MOODR no longer had a power advantage over MEDR (in fact had substantially less power) for the same location pattern as in Table 6.

When sample sizes were unequal and group locations were different, the power of all tests depended on the pattern of location parameters. MOODR was by far the most affected by the pattern of differences, with virtually no power in the most extreme case (smallest samples with nonzero location parameters and largest with zero location parameters), while sometimes having the highest power with the situation reversed. In contrast, MEDR maintained respectable power for all location patterns (See Tables 11 and 12). MEDBON displayed low power when sample sizes were small, especially with five groups (10 comparisons). Power was higher with larger sample sizes, but still generally trailed the other two procedures. Many other scenarios were examined. These results are available at www.uncg.edu/ sjricht2/Research.html. 
Table 13. $P$-values for pairwise comparisons.

\begin{tabular}{ccccccc} 
Comparison & Median & \multicolumn{5}{c}{ Procedure } \\
& difference & MEDR & MOODR & MEDUR & MOODUR & TUKEY \\
1vs2 & 85.0 & 0.950 & 1.000 & 0.794 & 0.974 & 0.985 \\
$1 \mathrm{vs} 3$ & 55.5 & 0.996 & 0.566 & 0.834 & 0.534 & 0.605 \\
$2 \mathrm{vs} 3$ & 140.5 & 0.691 & 0.295 & 0.645 & 0.345 & 0.372
\end{tabular}

Table 14. Average times to complete an interview for four interviewers.

Interviewer
1
2
3
4

Average time (min.)
$\begin{gathered}10.0,25.0,40.1,29.2,4.1 \\ 15.0,5.2,55.3,15.1,23.2 \\ 19.1,25.4,8.3 \\ 5.1,9.2,14.1\end{gathered}$

$\begin{array}{cc}\text { Median } & \text { Mean } \\ 25.0 & 21.6 \\ 15.1 & 22.8 \\ 19.1 & 17.6 \\ 9.2 & 9.5\end{array}$

Table 15. $P$-values for pairwise comparisons.

$\begin{array}{ccccccc}\text { Comparison } & \begin{array}{c}\text { Median } \\ \text { difference }\end{array} & \text { MEDR } & \text { MOODR } & \text { MEDUR } & \text { MOODUR } & \text { TUKEY } \\ \text { 1vs2 } & 9.9 & 0.851 & 1.000 & 0.920 & 1.000 & 0.999 \\ 1 \mathrm{vs} 3 & 5.9 & 1.000 & 1.000 & 0.978 & 0.915 & 0.980 \\ 1 \mathrm{vs} 4 & 15.8 & 0.211 & 0.450 & 0.525 & 0.362 & 0.666 \\ 2 \mathrm{vs} 3 & 4.0 & 1.000 & 1.000 & 1.000 & 0.915 & 0.961 \\ 2 \mathrm{vs} 4 & 5.9 & 1.000 & 0.450 & 0.978 & 0.362 & 0.607 \\ 3 \mathrm{vs} 4 & 9.9 & 0.851 & 0.824 & 0.920 & 0.915 & 0.900\end{array}$

Power Advantages of Median-based Procedures

The power of the median-based procedures was compared to that of the TukeyKramer procedure using means. For normally distributed data and equal sample sizes, TUKEY always had higher power than the median-based procedures (See Tables 4-6). However, with unequal sample sizes, the median based procedures often had higher power even for normally distributed data (See Tables 10, 11 and 12). This may not be surprising, since the Tukey-Kramer procedure has been shown to be conservative for unequal sample sizes (Hayter, 1984). For nonnormally distributed data, the median-based procedures often had higher power, especially with larger sample sizes.
Conclusion

The maximum median difference test (MEDR) is recommended as a robust pairwise comparison procedure when strong control of FWE is desired. The maximum Mood difference test (MOODR) is not recommended, due to poor power properties, especially for unequal sample sizes. Likewise, the procedure of using separate median difference tests with a Bonferroni adjustment (MEDBON) generally had less power and no power in some cases with small sample sizes. Tukey's HSD (TUKEY) is preferred when groups have small and equal samples sizes $(n=5)$, even for nonnormal data, and also with normal data, regardless of the sample size. In all other cases, the maximum median difference test (MEDR) is preferred. With nonnormal data and large $(n \geq 20)$ equal 
sample sizes, and in all cases with unequal sample sizes, MEDR had higher power than TUKEY. MEDR never performed poorly with regard to power, and was often much more powerful than the other median-based procedures considered.

\section{Example 1}

The first example is based on the data in the Introduction (See Table 1.) Table 13 gives $p$ values for the three pairwise comparisons, for the MEDR, MEDUR, MOODR, MOODUR and TUKEY procedures. Notice that the Mood tests yield the most evidence for a difference between months two and three. This is an example of a scenario studied in the simulations, namely small samples with differences between all pairs, with larger differences associated with the larger samples, a case where the Mood tests often had the highest power.

Example 2:

Consider data reported by Gibbons (1985, p. 202) in Table 14. The data represent average times spent to complete an interview for four interviewers.

It is desired to test if there is evidence that certain interviewers tend to have longer interview times. Table 15 gives $p$-values for the six pairwise comparisons. Here MEDR provides the strongest evidence of location difference between the pair with the largest observed difference, interviewers 1 and 4. Resampling Stats code for calculating the permutation $p$ values in this example is provided in the Appendix.

\section{References}

Gibbons, J. D. (1985). Nonparametric Methods for Quantitative Analysis, $2^{\text {nd }}$ edition. Columbus, OH: American Sciences Press, Inc.

Hayter, A. J. (1984). A proof of the conjecture that the Tukey-Kramer multiple comparisons procedure is conservative. Annals of Statistics, 12, 61-75.

Higgins, J. J. (2004). Introduction to Modern Nonparametric Statistics. Pacific Grove, CA: Brooks/Cole.
Hochberg Y. \& Tamhane, A. C. (1987). Multiple Comparison Procedures. New York: John Wiley and Sons.

Keller-McNulty, S. \& Higgins, J. J. (1987). Effect of tail weight and outliers on power and type-I error of robust permutation tests for location. Communications in Statistics: Simulation and Computation, 16(1):17-36.

Kramer, C. Y. (1956). Extension of multiple range tests to group means with unequal numbers of replications. Biometrics, 12, 309-310.

Levy (1979). Pairwise Comparisons associated with the $\mathrm{k}$ independent sample median test. The American Statistician, 33(3), 138-139.

Linton, L. R., Edgington, E. S. \& Davies, R. W. (1989). A view of niche overlap amenable to statistical analysis. Canadian Journal of Zoology, 67, 55-60.

Manly, B. F. J. (1997). Randomization, Bootstrap and Monte Carlo Methods in Biology, $\left(2^{\text {nd }}\right.$ ed.). London: Chapman \& Hall.

Miller, R. G. (1966). Simultaneous statistical inference. New York: McGraw-Hill.

Miller, R. G. (1981). Simultaneous statistical inference. ( $2^{\text {nd }}$ ed.). New York: Springer-Verlag.

Mood, A. M. (1950). Introduction to the Theory of Statistics. New York: McGraw-Hill.

Nemenyi, P. (1963). Distribution-free multiple comparisons. Unpublished doctoral dissertation, Princeton University, Princeton, NJ.

Petrondas, D. A. \& Gabriel, K. R. (1983). Multiple comparisons by rerandomization tests. Journal of the American Statistical Association, 78, 949-957.

Powell, G. L. \& Russell, A. P. (1984). The diet of the eastern short-horned lizard (Phrynosoma douglassi breviroste) in Alberta and its relationship to sexual size dimorphism. Canadian Journal of Zoology, 62, 428-440.

Powell, G. L. \& Russell, A. P. (1985). Growth and sexual size dimorphism in Alberta populations of the eastern short-horned lizard, Phrynosoma douglassi breviroste. Canadian Journal of Zoology, 63, 139-154.

Resampling Stats (2000). Resampling Stats Inc., Arlington, Virginia. 
Ryan, T. A. \& Ryan, T. A., Jr. (1980). K Independent sample median test. A letter to the Editor, The American Statistician, 34, 123.

Shaffer, J. P. (1995). Multiple hypothesis testing. Annual Review of Psychology, 46, 561-584.

Tukey, J. W. (1949). Comparing individual means in the analysis of variance. Biometrics, 5, 99-114.

\section{Appendix}

Below is Resampling Stats ${ }^{\circledR}$ code to calculate the permutation $p$-values in Example 2. The program can be modified to handle different numbers of groups.

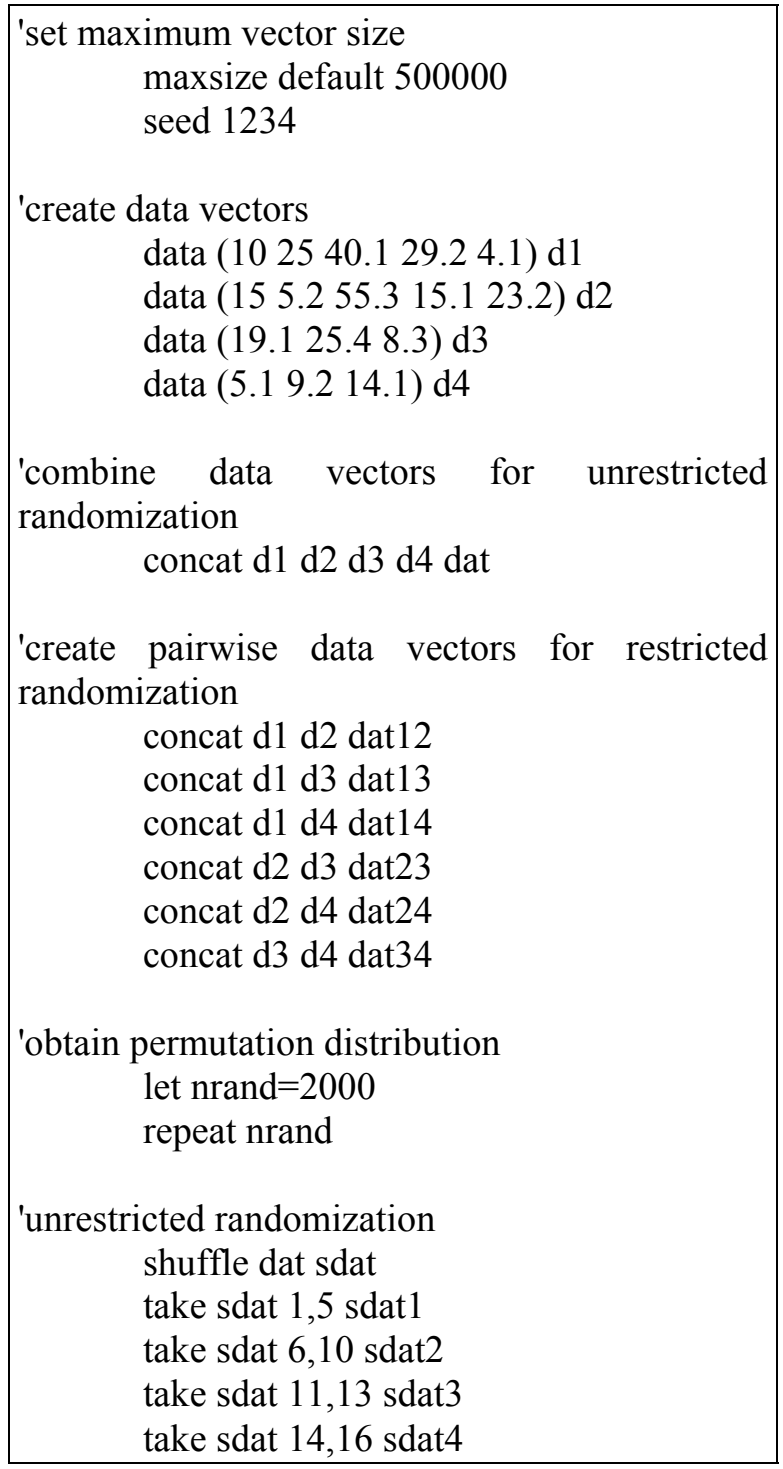

\begin{tabular}{|c|}
\hline 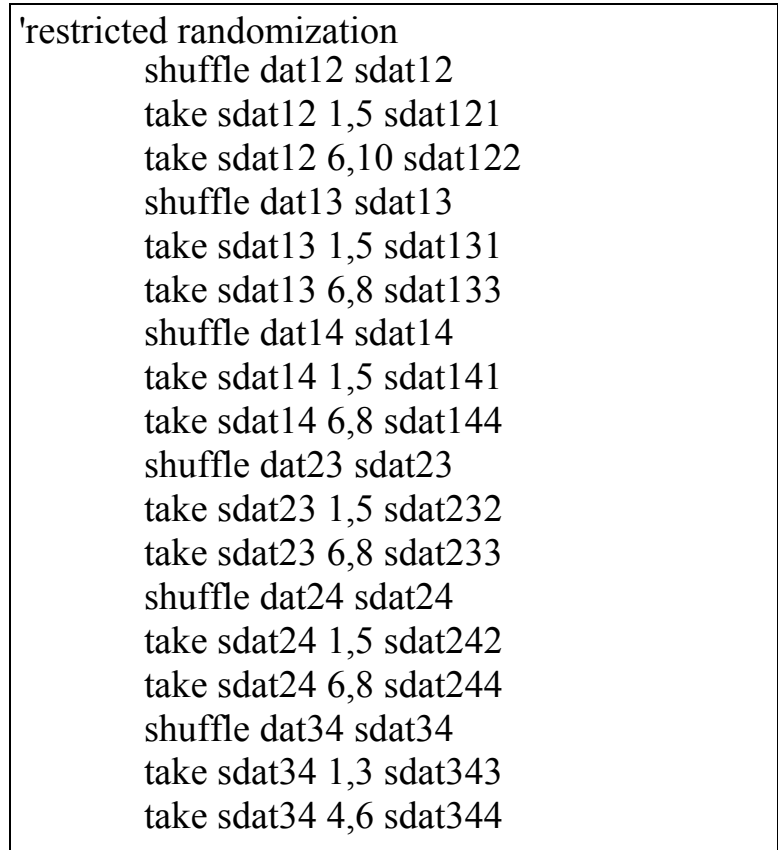 \\
\hline 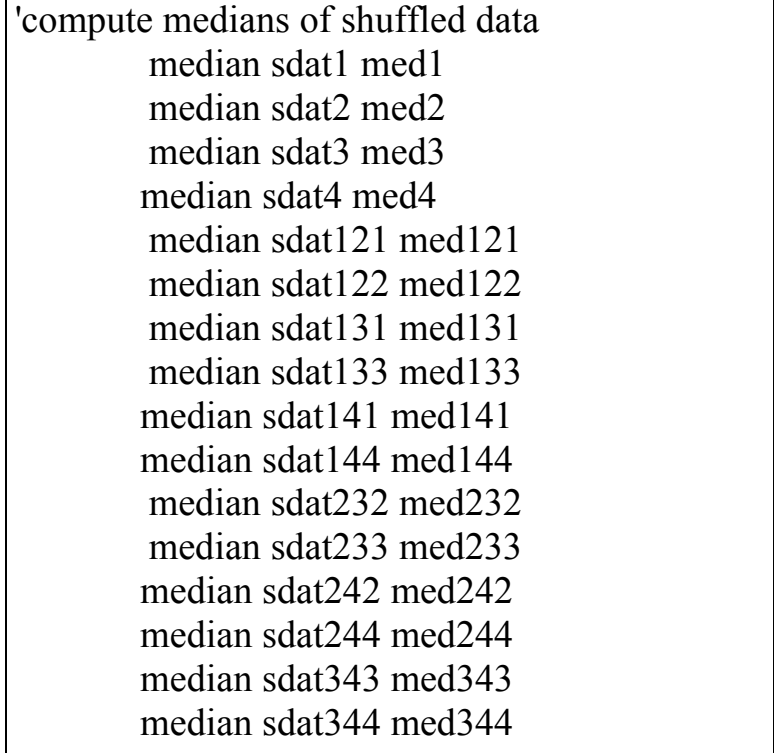 \\
\hline $\begin{array}{l}\text { 'compute median differences of shuffled data, } \\
\text { unrestricted randomization } \\
\text { subtract med } 1 \text { med } 2 \text { med } 12 \\
\text { subtract med } 1 \text { med } 3 \text { med } 13 \\
\text { subtract med } 1 \text { med } 4 \text { med } 14 \\
\text { subtract med } 2 \text { med } 3 \text { med } 23 \\
\text { subtract med } 2 \text { med } 4 \text { med } 24 \\
\text { subtract med } 3 \text { med } 4 \text { med34 }\end{array}$ \\
\hline $\begin{array}{l}\text { 'create one vector, take absolute values } \\
\text { concat med12 med13 med14 med23 } \\
\text { med24 med34 }\end{array}$ \\
\hline
\end{tabular}




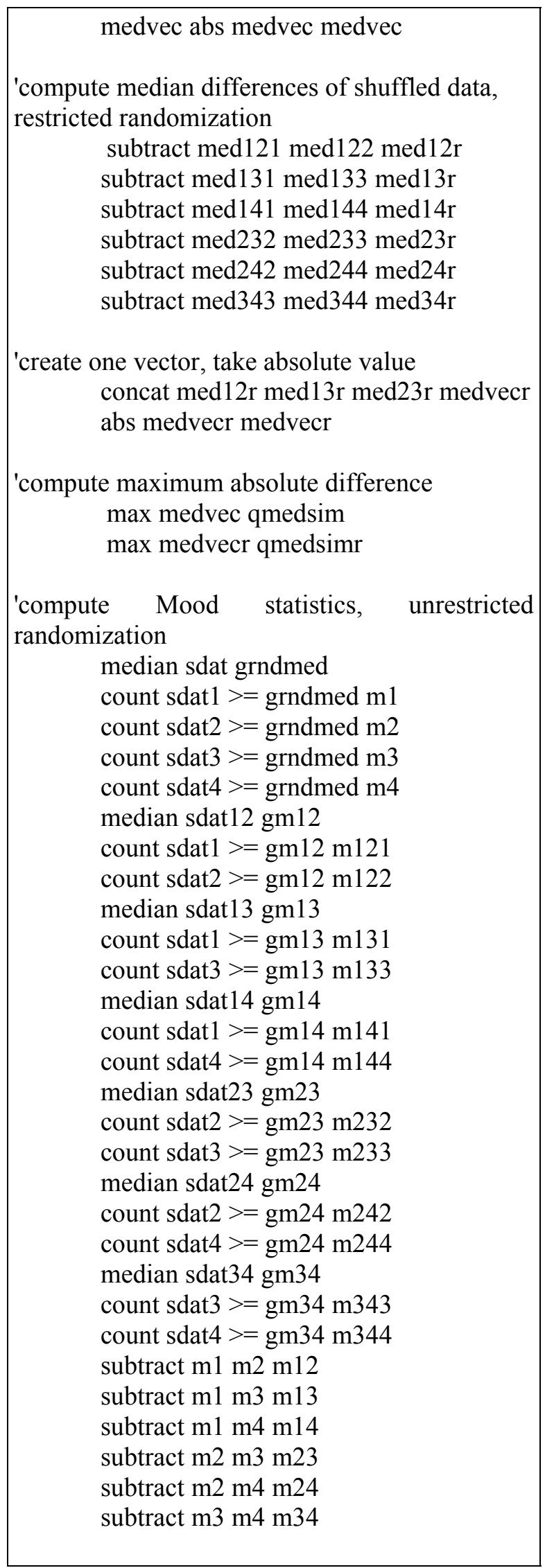

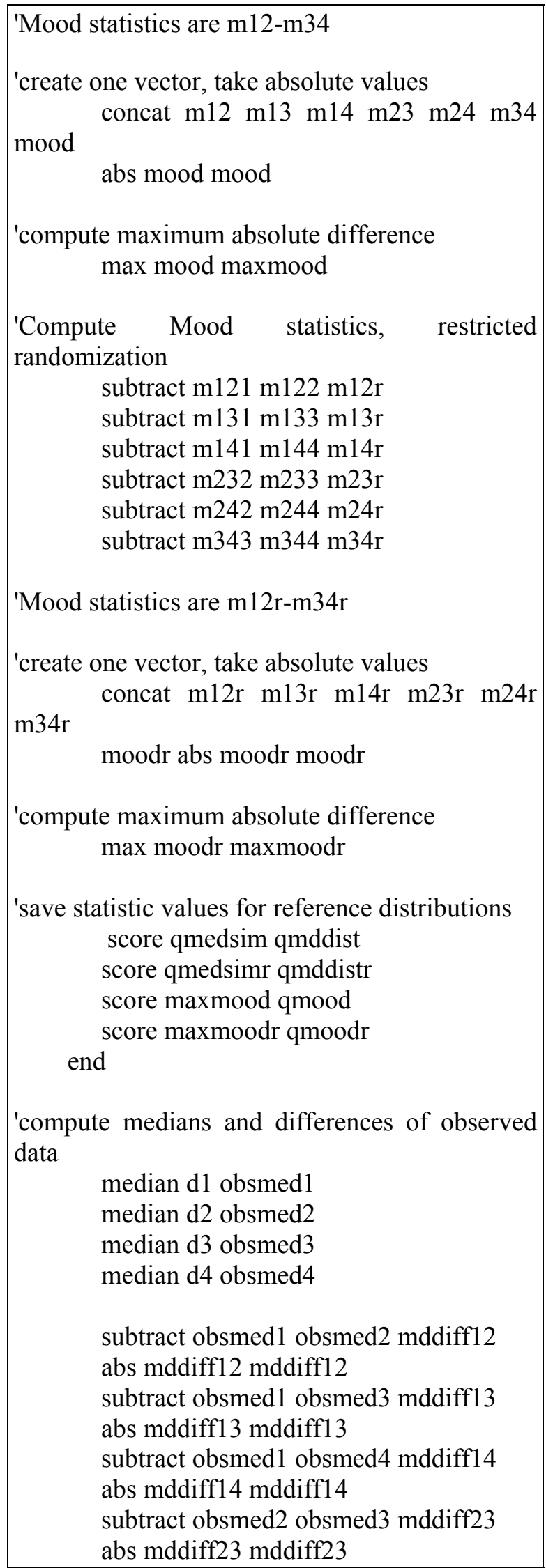




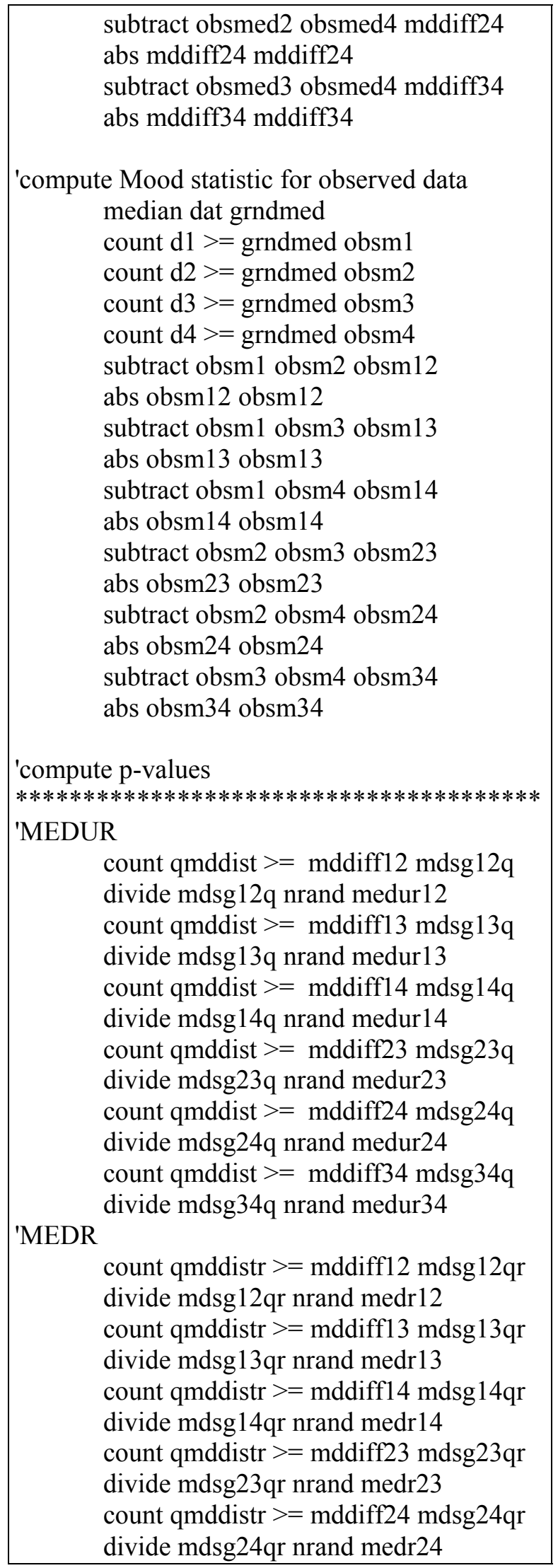

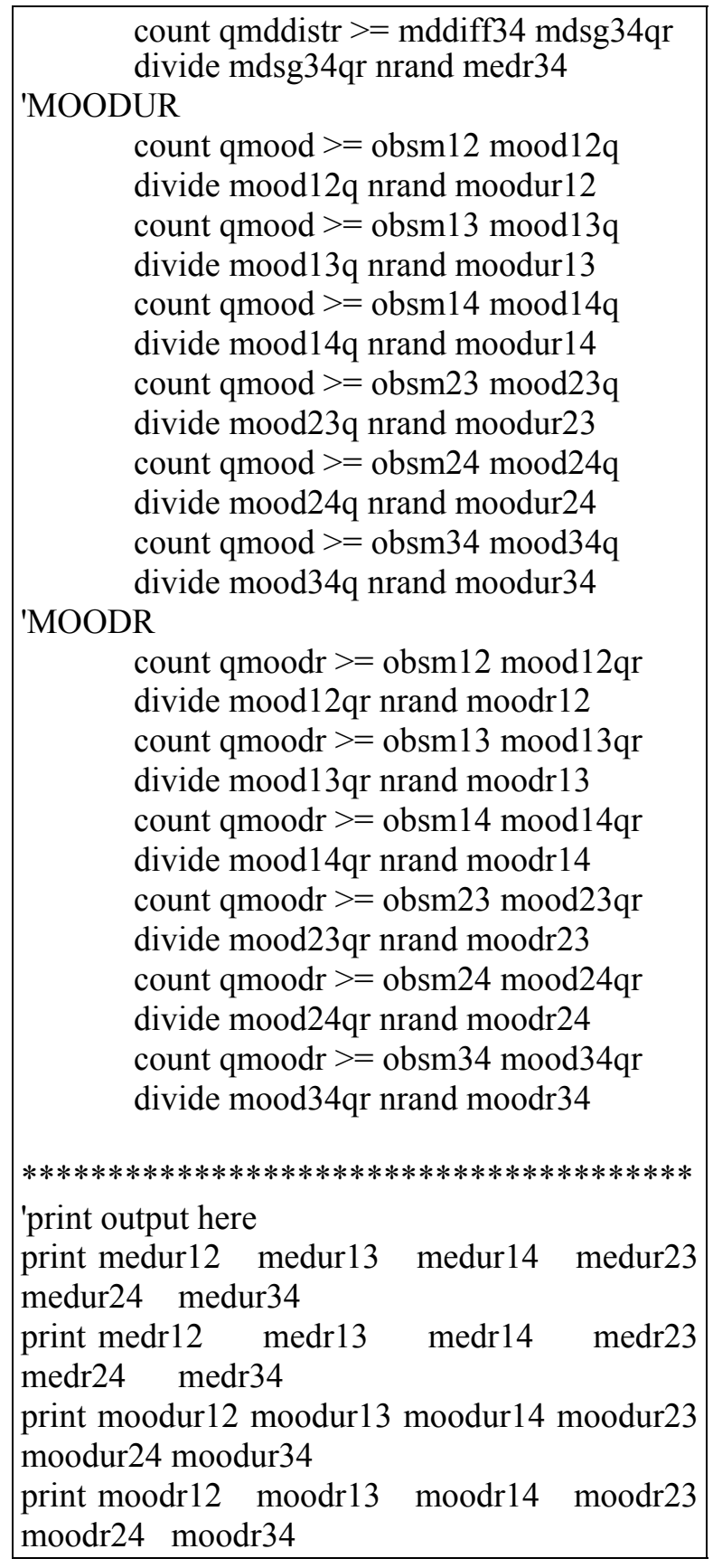

